\title{
LRRK2 G2019S Mutation: Prevalence and Clinical Features in Moroccans with Parkinson's Disease
}

\author{
Ahmed Bouhouche, ${ }^{1,2}$ Houyam Tibar, ${ }^{2}$ Rafiqua Ben El Haj, ${ }^{1}$ Khalil El Bayad, \\ Rachid Razine, ${ }^{3}$ Sanaa Tazrout, ${ }^{2}$ Asmae Skalli, ${ }^{1}$ Naima Bouslam, ${ }^{2}$ Loubna Elouardi, \\ Ali Benomar, ${ }^{1,2}$ Mohammed Yahyaoui, ${ }^{1,2}$ and Wafa Regragui ${ }^{1,2}$ \\ ${ }^{1}$ Research Team in Neurology and Neurogenetics, Medical School and Pharmacy, Mohammed V University, Rabat, Morocco \\ ${ }^{2}$ Department of Neurology and Neurogenetics, Specialties Hospital, Rabat, Morocco \\ ${ }^{3}$ Laboratory of Public Health, Medical School and Pharmacy, Mohammed V University, Rabat, Morocco
}

Correspondence should be addressed to Ahmed Bouhouche; a.bouhouche@um5s.net.ma

Received 9 December 2016; Accepted 23 March 2017; Published 30 March 2017

Academic Editor: Daniel Berwick

\begin{abstract}
Copyright ( 2017 Ahmed Bouhouche et al. This is an open access article distributed under the Creative Commons Attribution License, which permits unrestricted use, distribution, and reproduction in any medium, provided the original work is properly cited.
\end{abstract}

\begin{abstract}
Background. The LRRK2 G2019S mutation is the most common genetic determinant of Parkinson's disease (PD) identified to date. This mutation, reported in both familial and sporadic PD, occurs at elevated frequencies in Maghreb population. In the present study, we examined the prevalence of the G2019S mutation in the Moroccan population and we compared the motor and nonmotor phenotype of G2019S carriers to patients with idiopathic Parkinson's disease. Methods. 100 PD patients were assessed for motor and nonmotor symptoms, current medication, and motor complication including motor fluctuations and dyskinesia. The LRRK2 G2019S mutation was investigated by direct sequencing in patients and ethnically matched controls, all of Moroccan origin. Results. Among the 100 PD Moroccan patients, 41 (41\%) were carriers of the G2019S mutation. The mutation frequency was higher among probands with autosomal dominant inheritance (76\%) than among sporadic ones (28\%). Interestingly, G2019S mutation was also found in 5\% of control individuals. Clinically, patients carrying the G2019S mutation have more dystonia $(\mathrm{OR}=4.6, p=0.042)$ and more sleep disorders ( $\mathrm{OR}=2.4, p=0.045)$ than noncarriers. Conclusions. The LRRK2 G2019S prevalence in Morocco is the highest in the world reported to date. Some clinical features in G2019S carriers such as dystonia and sleep disturbances are worth noting.
\end{abstract}

\section{Introduction}

Parkinson's disease (PD) is the second most common neurodegenerative disorder after Alzheimer's disease affecting approximately $1-2 \%$ of the population over 60 years and $4 \%$ above 85 years [1]. It is clinically characterized by rigidity, bradykinesia, tremor, and postural instability. Other clinical features as dementia and depression can be added to this clinical array $[2,3]$. Pathologically it is identified by a selective degeneration of dopaminergic neurons in the substantia nigra in the midbrain and eventually the presence of Lewy bodies in the surviving neurons $[4,5]$. The etiology of PD is likely to be multifactorial involving complex interactions between genetic and environmental factors, but the exact molecular mechanism underlying the pathogenesis of the disease remains obscure. In the past 17 years, genetic studies of PD families consolidate the hypothesis that PD has a significant genetic component. Indeed, 14 genes have been described for Mendelian PD so far [6, 7]. Among them, there are at least three confirmed genes responsible for the autosomal dominant form of PD: SNCA (PARK1/4), LRRK2 (PARK8), and VPS35 (PARK17).

Mutations in LRRK2 gene are the most frequently reported monogenic cause of $\mathrm{PD}$ and are common in both early and late-onset PD, occurring in both familial and sporadic PD patients with a wide variety of clinical and pathological features and a variable frequency depending on ethnic origin [8]. Among these mutations, the glycine to serine substitution (G2019S), located within the protein kinase domain encoded by exon 41 , is the most common and was estimated by the international LRRK2 consortium to represent $1 \%$ of sporadic and $4 \%$ of familial PD patients 
worldwide [9]. Intriguingly, the frequency of this mutation varies greatly among ethnic groups and geographic origins. In fact, the highest frequencies were observed in North African countries with 30-40\% and Ashkenazi Jews with 10-30\% $[10,11]$. In Europe, the frequency of G2019S mutation appears to be relatively higher in southern countries particularly in Portugal and Spain with 2-14\% of PD cases, than in northern countries with $0-3 \%$ [9-13] suggesting a European northsouth gradient. The presence of G2019S in PD patients is very rare in Asian populations with a frequency less than $0.1 \%$ in China, Japan, Korea, and India, whereas it can reach $1-3 \%$ in white North American population $[8,10,11$, 14]. However, none of black PD patients from Nigeria and South Africa seems to carry the G2019S mutation $[15,16]$. Among apparently healthy controls, the highest frequency of the G2019S mutation has been reported in North Africa with 3.3\% in Berbers of Morocco, 2.13\% in Algerians, $1.57 \%$ in Tunisians, and $1.32 \%$ in Libyans [17]. This frequency is estimated at 2\% in Ashkenazi Jews [18] and is reported as very rare or absent in other populations [9]. Occurrence of the G2019S mutation in patients with PD and healthy subjects suggests reduced penetrance, which has been shown to vary according to ethnic origin [12,19-22]. This variability in penetrance suggests that other genetic or environmental factors are involved in the pathogenesis of the disease.

Clinically, G2019S mutation carriers develop a very similar PD disease to noncarriers, including the development of motor symptoms and cognitive impairment $[9,23]$, but some differences could be observed even within the same family [24]. Homozygous carriers of G2019S mutation are rare, mostly reported in North African populations where the rate of consanguineous marriages is high. These patients do not show differences in clinical features compared with heterozygous carriers [25-30].

The present study aims to estimate the prevalence of the G2019S mutation of the LRRK2 gene in the Moroccan population and to assess the motor and nonmotor phenotype of G2019S mutation carriers and noncarriers.

\section{Subjects and Methods}

A total of 100 unrelated PD patients were recruited consecutively from the outpatient clinic of the Neurological Department at University Hospital Ibn Sina of Rabat from October 2013 to June 2015. One hundred healthy individuals were recruited from the National Blood Transfusion Institute of Rabat and were used as controls. Their mean age was 58.59 $( \pm 8.65)$ and 51 of them were males. Patients and controls provided a written informed consent and the study was approved by the ethic committee of the Medical School of Rabat (CERB).

2.1. Clinical Evaluation. Diagnosis of PD was made by the same neurologist using the United Kingdom Parkinson's Disease Society Brain Bank criteria [31]. Patients were submitted to a structured clinical interview including demographic data, date of onset, disease duration, motor phenotype subtype, the presence of dystonia in the early disease course, motor fluctuations and dyskinesia, nonmotor symptoms, and current medication. Motor symptoms were assessed using the Unified Parkinson's Disease Rating Scale part III and Hoehn and Yahr stage during ON condition. The LEDD (levodopa Equivalent Daily Dose) was calculated based on a previously published algorithm combining dopamine agonist daily dose with levodopa daily dose [32]. We classified the motor phenotype as tremor-dominant, akineticrigid, or mixed, and for the purposes of analysis owing to low figures, we included akinetic-rigid phenotype in mixed group. Nonmotor symptoms scales used are the Pittsburgh sleep QI for sleep disturbances, the Epworth Sleepiness Scale for excessive daytime sleepiness, the SCOPA autonomic questionnaire for dysautonomia, the DN4 questionnaire for neuropathic pain, the Hamilton and the Montgomery and Asberg Depression Rating Scale (MADRS) for depressive complaints, and the Arab version of the MMSE for cognitive impairment (patients with scores below 21 were excluded to avoid reliability concerns in their answers relative to the scales of the questionnaire). For simplification, we recorded nonmotor symptoms as absent or present for constipation, urinary urgency, orthostatic vertigo, pain, hallucinations, memory complaints, and sleep disturbances.

Otherwise, all control individuals have no family history of neurological disease but have not been clinically assessed for the presence of PD.

2.2. Genetic Analysis. A pedigree was established for all patients and the mode of inheritance was classified as "familial" if at least one relative was reported with a diagnosis of PD (FPD) and as autosomal recessive or dominant based on the presence or absence of consanguinity. The remaining patients were classified as "sporadic" (SPD).

Genomic DNA was extracted from peripheral blood leukocytes using Isolate II Genomic DNA kit from Bioline. The G2019S mutation of the LRRK2 gene was performed by direct sequencing. Briefly, a 378 bp LRRK2 exon 41 fragment was PCR amplified as described previously [33]. The PCR products were sequenced using Big Dye Terminator Cycle Ready Reaction 3.1 Kits and an ABI 3130xl automated sequencer, and sequence chromatograms were analyzed using SeqScape 2.1 software (Applied Biosystems, Foster City, CA).

2.3. Statistical Analysis. Demographic and clinical variables between G2019S-carriers and noncarriers were compared using parametric and nonparametric tests as appropriate using SPSS 13.0 software. Quantitative data were expressed in mean \pm standard deviation (SD) or median and interquartile range and were compared using $t$-test or Mann-Whitney test. Categorical variables were expressed as numbers and percentages and were compared using Chi-square test. The relationship between G2109S mutation and the clinical symptoms was analyzed by means of logistic regression adjusting for age, sex, and disease duration on univariate and multivariate analysis. In the multivariate analysis model, we introduced variables that had a $p \leq 0.3$ and we forced this analysis for Hoehn and Yahr score for its importance in the disease evolution. $p$ value $<0.05$ was considered as statistically 
TABLE 1: Demographic features of LRRK2 G2019S carrier and noncarrier patients.

\begin{tabular}{|c|c|c|c|c|}
\hline Variables & $\begin{array}{l}\text { All patients } \\
\quad(n=100)\end{array}$ & $\begin{array}{c}\text { G2109S carriers } \\
\quad(n=41)\end{array}$ & $\begin{array}{l}\text { G2019S noncarriers } \\
\qquad(n=59)\end{array}$ & $p$ value \\
\hline Age at exam (years) ${ }^{\mathrm{a}}$ & $60.93 \pm 11.07$ & $60.32 \pm 11.83$ & $61.35 \pm 10.59$ & 0.649 \\
\hline Age at onset (years) ${ }^{\mathrm{a}}$ & $53.9 \pm 11.54$ & $52.15 \pm 11.28$ & $55.12 \pm 11.65$ & 0.207 \\
\hline Disease duration $\left(\right.$ years) ${ }^{\mathrm{b}}$ & $6[2.25-10]$ & 7 [4-13] & $5[2-7]$ & 0.035 \\
\hline Gender $(\text { male })^{c}$ & 56 & $19(46.3)$ & $37(62.7)$ & 0.105 \\
\hline
\end{tabular}

Data are ${ }^{a}$ mean \pm standard deviation, ${ }^{b}$ median [interquartile range], and ${ }^{c}$ numbers (percentage).

TABLE 2: Prevalence of G2019S mutations among 100 Moroccan PD patients.

\begin{tabular}{|c|c|c|c|c|c|c|c|}
\hline \multirow{2}{*}{$\begin{array}{l}\text { Form } \\
\text { Inheritance }\end{array}$} & \multicolumn{4}{|c|}{ Familial } & \multicolumn{3}{|c|}{ Sporadic cases } \\
\hline & DFPD & RFPD & $\mathrm{ND}$ & Total & Inbred & Not inbred & Total \\
\hline Patients & $29(29)$ & $2(2)$ & $2(2)$ & $33(33)$ & $10(10)$ & $57(57)$ & $67(67)$ \\
\hline G2019S carriers & $22(76)$ & $0(0)$ & $0(0)$ & $22(67)$ & $1(10)$ & $18(32)$ & $19(28)$ \\
\hline
\end{tabular}

Data are number (percentage). DFPD = dominant familial Parkinson's disease, RFPD = recessive familial Parkinson's disease, and ND $=$ inheritance not determined.

significant. For multiple testing, we corrected the $p$ value by the Bonferroni method.

\section{Results}

We examined 100 patients with PD, 56 of whom were males and 44 were females (Table 1). The mean age at exam was $60.93( \pm 11.07)$ and the mean age at onset was 53.9 years $( \pm 11.54)$. Comparison of demographic features between the G2019S carriers and noncarriers (Table 1) showed no significant difference except for the disease duration; G2019S carriers have the longest disease duration $(p=0.03)$.

3.1. Genetic Aspects. Sixty-seven out of 100 patients were sporadic cases $(67 \%)$ and 33 had a positive family history of $\mathrm{PD}$ (33\%). Ten of the 67 SPD patients were from consanguineous marriages (Table 2). Among the 33 FPD patients, 29 had dominant inheritance (DFPD), 2 had recessive inheritance (RFPD), and in 2 patients the mode of inheritance could not be specified (parents were consanguineous and one of them is with PD). The LRRK2 G2019S substitution was found in 41 of 100 (41\%, 95\% CI 31.4-50.3) PD patients, 37 of whom were heterozygous and 4 were homozygous. The G2019S prevalence increased to $67 \%$ (95\% CI 48.11-81.45) for FPD patients, with $76 \%$ of patients (95\% CI 56.07-88.98) having a DFPD, $0 \%$ of patients ( 0 of 2 ) having RFPD, and $0 \%$ of patients ( 0 of 2) having an unspecified mode of inheritance. The prevalence reaches only $28 \%$ (95\% CI $18.35-40.88$ ) for SPD patients with $32 \%$ of patients (95\% CI 20.27-45.38) without consanguinity and $10 \%$ of patients (95\% CI 1.79-40.41) with consanguinity (Table 2). Interestingly, there were five control individuals heterozygous for the G2019S mutation among the 100 tested (5\%, 95\% CI 1-10).

3.2. Clinical Features. Motor and nonmotor symptoms of all patients are given in Table 3 . The initial symptom of the disease and the clinical phenotype were significantly different between LRRK2 G2019S carriers and noncarriers
( $p=0.019$ and $p=0.012$, resp.). LRRK2 G2019S carriers have less tremor than noncarriers do as first symptom $(26.8 \%$ versus $52.5 \%$, corrected $-p=0.03$ ) and exhibited less of the tremor-dominant phenotype than noncarriers (corrected- $p=$ $0.009)$. They also have more dystonia $(p=0.011)$ and more dyskinesia $(p=0.002)$ and take a higher dose (up to $200 \mathrm{mg}$ ) of dopaminergic drugs $(p=0.002)$. Gait disturbances, postural instability, motor fluctuations, UPDRS III score, and H\&Y scale during $\mathrm{ON}$ state are similar between both groups. Concerning nonmotor symptoms, LRRK2 G2019S carriers have more sleep complaints than noncarriers do $(p=0.046)$, while they show the same rates of psychiatric symptoms, constipation, urinary urgency, and orthostatic vertigo. There is a trend to more cognitive impairment in LRRK2 G2019S carriers than noncarriers but the difference is not significant $(p=0.059)$.

The relationship between G2109S mutation and the clinical symptoms using logistic regression by adjusting for age, sex, and disease duration is shown in Table 4. LRRK2 G2019S mutation is associated with more dystonia $(\mathrm{OR}=4.655, p$ $=0.042)$ and sleep complaints $(\mathrm{OR}=2.4, p=0.045)$ but less tremor $(\mathrm{OR}=0.3, p=0.011)$. Nonetheless, while $L R R K 2$ G2019S carriers have more levodopa-induced dyskinesia ( $p$ $=0.002)$, the statistical significance lacked on multivariate analysis $(\mathrm{OR}=1.965, p=0.217)$.

\section{Discussion}

The international $L R R K 2$ consortium reported a worldwide frequency of $L R R K 2$ G2019S mutation of $1 \%$ in sporadic PD and $4 \%$ in familial PD [9]. Although the highest frequency of the G2019S mutation has been recorded in North Africa, no study on the prevalence of this mutation in the Moroccan population has been conducted until now. Previous studies have used small sample size of Moroccan patients, the majority of whom were living outside Morocco. In our cohort of $100 \mathrm{PD}$ patients of Moroccan origin, the overall mutation frequency of G2019S is $41 \%$ (95\% CI 31.4-50.3). Among probands with autosomal dominant mode of inheritance, this 
TABLE 3: Clinical features of G2019S carrier and noncarrier patients.

\begin{tabular}{|c|c|c|c|}
\hline & $\begin{array}{c}\text { G2109S carriers } \\
\quad(n=41)\end{array}$ & $\begin{array}{l}\text { G2019S noncarriers } \\
\qquad(n=59)\end{array}$ & $p$ value \\
\hline Initial symptom & & & 0.019 \\
\hline Akinesia $^{\mathrm{a}}$ & $14(34.1)$ & $9(15.3)$ & \\
\hline Tremor $^{\mathrm{a}}$ & $11(26.8)$ & $31(52.5)$ & \\
\hline Tremor and akinesia ${ }^{a}$ & $16(39)$ & $19(32.2)$ & \\
\hline Clinical phenotype & & & 0.012 \\
\hline Akinetic-rigid $^{\mathrm{a}}$ & $10(24.4)$ & $10(16.9)$ & \\
\hline Tremor-dominant $\mathrm{t}^{\mathrm{a}}$ & $7(17.1)$ & $27(45.8)$ & \\
\hline Mixed $^{\mathrm{a}}$ & $24(58.5)$ & $22(37.3)$ & \\
\hline Dystonia $^{\mathrm{a}}$ & $9(21.9)$ & $3(5.1)$ & 0.011 \\
\hline Gait impairment $^{\mathrm{a}}$ & $21(51.2)$ & $23(39)$ & 0.225 \\
\hline Postural instability ${ }^{\mathrm{a}}$ & $17(41.5)$ & $29(49.2)$ & 0.448 \\
\hline UPDRS III ON ${ }^{\mathrm{b}}$ & $11[6.5-19]$ & $11[6-21]$ & 0.858 \\
\hline H-Y score ${ }^{b}$ & $2[1-4]$ & $3[1-4]$ & 0.875 \\
\hline $\operatorname{LEDD}^{\mathrm{b}}$ & $727.9[500-1100]$ & $500[300-800]$ & 0.002 \\
\hline Fluctuations $^{\mathrm{a}}$ & $25(61)$ & $29(49.2)$ & 0.243 \\
\hline Dyskinesia $^{\mathrm{a}}$ & $23(56.1)$ & $15(25.4)$ & 0.002 \\
\hline Urinary dysfunction $^{a}$ & $30(73.2)$ & $48(82.8)$ & 0.253 \\
\hline Constipation $^{\mathrm{a}}$ & $21(51.2)$ & $31(52.5)$ & 0.896 \\
\hline Orthostatic hypotension $^{a}$ & $16(39.0)$ & $29(49.2)$ & 0.318 \\
\hline Pain $^{\mathrm{a}}$ & $22(53.7)$ & $31(52.5)$ & 0.912 \\
\hline Psychiatric disorders ${ }^{a}$ & $25(61.0)$ & $28(47.5)$ & 0.184 \\
\hline Cognitive disorders $^{\mathrm{a}}$ & $26(63.4)$ & $26(44.1)$ & 0.059 \\
\hline Sleep disorders ${ }^{\mathrm{a}}$ & $22(53.7)$ & $19(33.3)$ & 0.046 \\
\hline
\end{tabular}

Data are ${ }^{\mathrm{a}}$ number (percentage) and ${ }^{\mathrm{b}}$ median [interquartile range]. Significant $p$ values are in bold.

TABLE 4: Logistic regression model of the association between G2019S mutation and PD clinical features adjusted for gender, age, and disease duration.

\begin{tabular}{|c|c|c|c|}
\hline & $\mathrm{OR} / \beta$ & CI 95\% & $p$ value \\
\hline Initial symptom & 0.305 & $0.123-0.758$ & 0.011 \\
\hline \multicolumn{4}{|l|}{ Tremor $^{\mathrm{a}}$} \\
\hline \multicolumn{4}{|l|}{ Akinesia + mixed $^{\mathrm{a}}$} \\
\hline Clinical phenotype & 0.261 & $0.096-0.708$ & 0.008 \\
\hline \multicolumn{4}{|l|}{ Tremor-dominant ${ }^{\mathrm{a}}$} \\
\hline \multicolumn{4}{|c|}{ Akinetic-rigid + mixed $^{\mathrm{a}}$} \\
\hline Dystonia $^{\mathrm{a}}$ & 4.655 & $1.058-20.48$ & 0.042 \\
\hline H-Y score ${ }^{b}$ & -0.104 & $-0.666-458$ & 0.717 \\
\hline $\operatorname{LEDD}^{\mathrm{b}}$ & 52.57 & $-103.6-208.7$ & 0.509 \\
\hline Fluctuations $\mathrm{s}^{\mathrm{a}}$ & 0.606 & $0.206-1.782$ & 0.363 \\
\hline Dyskinesia $^{a}$ & 1.965 & $0.673-5.739$ & 0.217 \\
\hline Urinary dysfunction $^{\mathrm{a}}$ & 0.566 & $0.207-1.550$ & 0.268 \\
\hline Psychiatric disorders $^{a}$ & 2.023 & $0.856-4.782$ & 0.109 \\
\hline Cognitive disorders ${ }^{\mathrm{a}}$ & 1.892 & $0.812-4.409$ & 0.140 \\
\hline Sleep disorders ${ }^{\mathrm{a}}$ & 2.409 & $1.021-5.685$ & 0.045 \\
\hline
\end{tabular}

Data are ${ }^{\mathrm{a}}$ numbers (percentages) and ${ }^{\mathrm{b}}$ median [interquartile range]. $\mathrm{OR}=$ odds ratio and $\mathrm{CI}=$ confident interval. Significant $p$ values are in bold. 
value rises to about 76\% (95\% CI 56.07-88.98) and 28\% (95\% CI 18.35-40.88) among sporadic cases. Unexpectedly, these frequencies were higher than observed in PD patients from neighboring Maghreb countries such as Algeria and Tunisia $[10,11]$, representing the highest prevalence in the world reported to date for the G2019S mutation.

Among the 41 patients with G2019S mutation, four were homozygous carriers with different mode of inheritance, including two with autosomal dominant inheritance and two sporadic cases of which one comes from a consanguineous marriage. This could be due to the high prevalence of the mutation in the general population. Indeed, there were five heterozygous G2019S carriers among the 100 control individuals (5\%).

Clinically, most authors reported a similar phenotype between LRRK2 G2019S carriers and noncarriers [34, 35]. In our series, the phenotypes are overlapping but the LRRK2 subjects have less tremor. This finding is in line with some series [34] whereas others reported the tremor as a more common presenting feature in LRRK2 carriers [30, 36, 37]. Contrariwise, we found a nonsignificant increase of dyskinesia frequency as reported earlier [38], which can be explained by the higher LEDD recorded in this group. Moreover, LRRK2 G2019S carriers had a similar UPDRS III and H\&Y scores for longest disease duration $(7[4-13]$ versus 5 [2-7], $p=$ $0.035)$ compared to noncarriers. It can reflect in some extent a slower disease evolution in LRRK2 G2019S patients. In the same line, Healy et al. [9] reported longer latency between disease onset and first fall among carriers compared to noncarriers. However, longitudinal follow-up is needed to compare disease course between the two groups.

Furthermore, dystonia is the most specific feature that characterizes LRRK2 G2019S carriers in our series with an ODDS ratio of 4.65. Dystonia is a well-defined symptom in early onset Parkinson's disease (EOPD) as reported by Kilarski et al. [39] in their systematic review and UK-based study of EOPD. The high frequency in our study can be explained somehow by the overall young age of onset of Parkinson's disease in Moroccan patients [40], but there was no statistically difference in age of onset between LRRK2 G2019S carriers and noncarriers ( $p=0.207)$.

Otherwise, LRRK2 G2019S patients exhibited more sleep complaints in our series. This feature is in line with other series that reported more sleep onset insomnia, few or no rapid eye behavior disorders, and less nocturia in LRRK2 G2019S carriers [41-43].

Other reports described more specific clinical features in $L R R K 2$ carriers such as a lower limb onset [34], more hallucinations, behavioral disorders, and dopaminergic dysregulation syndrome $[36,38,44]$ but less cognitive impairment [34, 36, 45]. Kalia et al. [46] explained this phenomenon by the lack of Lewy bodies in some cases with LRRK2 G2019S mutation; the presence of Lewy bodies is strongly correlated to some nonmotor symptoms especially cognitive impairment and dementia. In our study, LRRK2 carriers have a trend to more cognitive impairment that was not confirmed in the logistic regression model. Future studies with wider cohorts are required to determine the cognitive profile of $L R R K 2$ carriers.

\section{Conclusions}

Our study showed that Morocco has the highest reported prevalence of the G2019S mutation in the world, with a mutation frequency of $41 \%$ overall and $76 \%$ for patients with autosomal dominant mode of inheritance. Furthermore, G2019S carrier patients exhibit clinical features quite similar to noncarriers with some mild differences in particular more dystonia and more sleep complaints.

\section{Conflicts of Interest}

The authors declare that they have no conflicts of interest.

\section{Acknowledgments}

The authors are grateful to the patients for their participation in this study. They also gratefully acknowledge the National Center of Blood Transfusion of Rabat for providing the control samples. The study was supported by Novartis Pharma Maroc SA and the "Centre National de Recherche Scientifique et Technique" (CNRST) of "Ministère de l'Enseignement Supérieur, de la Recherche Scientifique et de la Formation des Cadres" (MESRSFC) of Morocco.

\section{References}

[1] F. Copped, "Genetics and epigenetics of Parkinson's disease," The Scientific World Journal, vol. 2012, Article ID 489830, 2012.

[2] B. Thomas and M. Flint Beal, "Parkinson's disease," Human Molecular Genetics, vol. 16, no. 2, pp. R183-R194, 2007.

[3] J. Jankovic, "Parkinson's disease: clinical features and diagnosis," Journal of Neurology, Neurosurgery and Psychiatry, vol. 79, no. 4, pp. 368-376, 2008.

[4] M. C. J. Dekker, V. Bonifati, and C. M. Van Duijn, "Parkinson's disease: piecing together a genetic jigsaw," Brain, vol. 126, no. 8, pp. 1722-1733, 2003.

[5] D. J. Moore, A. B. West, V. L. Dawson, and T. M. Dawson, "Molecular pathophysiology of Parkinson's disease," Annual Review of Neuroscience, vol. 28, pp. 57-87, 2005.

[6] V. Bonifati, "Genetics of Parkinson's disease-state of the art, 2013," Parkinsonism and Related Disorders, vol. 20, no. 1, pp. S23-S28, 2014.

[7] M. K. Lin and M. J. Farrer, "Genetics and genomics of Parkinson's disease," Genome Medicine, vol. 6, no. 6, article 48, 2014.

[8] P. Gómez-Garre, F. Carrillo, and P. Mir, "Prevalence and clinical features of LRRK2 mutations in patients with Parkinson disease," European Neurology Journal, vol. 2, pp. 1-7, 2010.

[9] D. G. Healy, M. Falchi, S. S. O'Sullivan et al., "Phenotype, genotype, and worldwide genetic penetrance of LRRK2-associated Parkinson's disease: a case-control study," The Lancet Neurology, vol. 7, no. 7, pp. 583-590, 2008.

[10] H. T. S. Benamer and R. De Silva, "LRRK2 G2019S in the North African population: a review," European Neurology, vol. 63, no. 6, pp. 321-325, 2010.

[11] S. Lesage, E. Patin, C. Condroyer et al., "Parkinson's diseaserelated LRRK2 G2019S mutation results from independent mutational events in humans," Human Molecular Genetics, vol. 19, no. 10, Article ID ddq081, pp. 1998-2004, 2010.

[12] J. Kachergus, I. F. Mata, M. Hulihan et al., "Identification of a novel LRRK2 mutation linked to autosomal dominant 
parkinsonism: evidence of a common founder across European populations," American Journal of Human Genetics, vol. 76, no. 4, pp. 672-680, 2005.

[13] M. Sierra, I. González-Aramburu, P. Sánchez-Juan et al., "High frequency and reduced penetrance of IRRK2 g2019S mutation among Parkinson's disease patients in Cantabria (Spain)," Movement Disorders, vol. 26, no. 13, pp. 2343-2346, 2011.

[14] H. F. Chien, T. R. Figueiredo, M. A. Hollaender et al., "Frequency of the LRRK2 G2019S mutation in late-onset sporadic patients with Parkinson's disease," Arquivos de Neuro-Psiquiatria, vol. 72, no. 5, pp. 356-359, 2014.

[15] N. Okubadejo, A. Britton, C. Crews et al., "Analysis of Nigerians with apparently sporadic Parkinson disease for mutations in LRRK2, PRKN and ATXN3," PLoS ONE, vol. 3, no. 10, Article ID e3421, 2008.

[16] S. Bardien, A. Marsberg, R. Keyser et al., "LRRK2 G2019S mutation: frequency and haplotype data in South African Parkinson's disease patients," Journal of Neural Transmission, vol. 117, no. 7, pp. 847-853, 2010.

[17] N. Change, G. Mercier, and G. Lucotte, "Genetic screening of the G2019S mutation of the LRRK2 gene in southwest European, North African, and sephardic Jewish subjects," Genetic Testing, vol. 12, no. 3, pp. 333-339, 2008.

[18] A. Orr-Urtreger, C. Shifrin, U. Rozovski et al., "The LRRK2 G2019S mutation in Ashkenazi Jews with Parkinson disease: is there a gender effect?" Neurology, vol. 69, no. 16, pp. 1595-1602, 2007.

[19] L. N. Clark, Y. Wang, E. Karlins et al., "Frequency of LRRK2 mutations in early- and late-onset Parkinson disease," Neurology, vol. 67, no. 10, pp. 1786-1791, 2006.

[20] S. Punia, M. Behari, S. T. Govindappa et al., "Absence/rarity of commonly reported LRRK2 mutations in Indian Parkinson's disease patients," Neuroscience Letters, vol. 409, no. 2, pp. 83-88, 2006.

[21] J. J. Ferreira, L. C. Guedes, M. M. Rosa et al., "High prevalence of LRRK2 mutations in familial and sporadic Parkinson's disease in Portugal," Movement Disorders, vol. 22, no. 8, pp. 1194-1201, 2007.

[22] S. Goldwurm, M. Zini, A. Di Fonzo et al., "LRRK2 G2019S mutation and Parkinson's disease: a clinical, neuropsychological and neuropsychiatric study in a large Italian sample," Parkinsonism and Related Disorders, vol. 12, no. 7, pp. 410-419, 2006.

[23] S. Belarbi, N. Hecham, S. Lesage et al., "LRRK2 G2019S mutation in Parkinson's disease: a neuropsychological and neuropsychiatric study in a large Algerian cohort," Parkinsonism and Related Disorders, vol. 16, no. 10, pp. 676-679, 2010.

[24] C. Schulte and T. Gasser, "Genetic basis of Parkinson's disease: inheritance, penetrance, and expression," Application of Clinical Genetics, vol. 4, pp. 67-80, 2011.

[25] W. C. Nichols, N. Pankratz, D. Hernandez et al., "Genetic screening for a single common LRRK2 mutation in familial Parkinson's disease," Lancet, vol. 365, no. 9457, pp. 410-412, 2005.

[26] S. Lesage, P. Ibanez, E. Lohmann et al., "G2019S LRRK2 mutation in French and North African families with Parkinson's disease," Annals of Neurology, vol. 58, no. 5, pp. 784-787, 2005.

[27] L. Ishihara, R. A. Gibson, L. Warren et al., "Screening for Lrrk2 G2019S and clinical comparison of Tunisian and North American Caucasian Parkinson's disease families," Movement Disorders, vol. 22, no. 1, pp. 55-61, 2007.
[28] L. Warren, R. Gibson, L. Ishihara et al., "A founding LRRK2 haplotype shared by Tunisian, US, European and Middle Eastern families with Parkinson's disease," Parkinsonism and Related Disorders, vol. 14, no. 1, pp. 77-80, 2008.

[29] S. Lesage, S. Belarbi, A. Troiano et al., "Is the common LRRK2 G2019S mutation related to dyskinesias in North African Parkinson disease?" Neurology, vol. 71, no. 19, pp. 1550-1552, 2008.

[30] M. M. Hulihan, L. Ishihara-Paul, J. Kachergus et al., "LRRK2 Gly2019Ser penetrance in Arab-Berber patients from Tunisia: a case-control genetic study," The Lancet Neurology, vol. 7, no. 7, pp. 591-594, 2008.

[31] A. J. Hughes, S. E. Daniel, L. Kilford, and A. J. Lees, "Accuracy of clinical diagnosis of idiopathic Parkinson's disease: a clinicopathological study of 100 cases," Journal of Neurology Neurosurgery and Psychiatry, vol. 55, no. 3, pp. 181-184, 1992.

[32] C. L. Tomlinson, R. Stowe, S. Patel, C. Rick, R. Gray, and C. E. Clarke, "Systematic review of levodopa dose equivalency reporting in Parkinson's disease," Movement Disorders, vol. 25, no. 15, pp. 2649-2653, 2010.

[33] J.-W. Cho, S.-Y. Kim, S.-S. Park, and B. S. Jeon, "The G2019S LRRK2 mutation is rare in Korean patients with Parkinson's disease and multiple system atrophy," Journal of Clinical Neurology (Korea), vol. 5, no. 1, pp. 29-32, 2009.

[34] R. N. Alcalay, A. Mirelman, R. Saunders-Pullman et al., "Parkinson disease phenotype in Ashkenazi jews with and without LRRK2 G2019S mutations," Movement Disorders, vol. 28, no. 14, pp. 1966-1971, 2013.

[35] A. Puschmann, "Monogenic Parkinson's disease and parkinsonism: clinical phenotypes and frequencies of known mutations," Parkinsonism and Related Disorders, vol. 19, no. 4, pp. 407-415, 2013.

[36] N. L. Khan, S. Jain, J. M. Lynch et al., "Mutations in the gene LRRK2 encoding dardarin (PARK8) cause familial Parkinson's disease: clinical, pathological, olfactory and functional imaging and genetic data," Brain, vol. 128, no. 12, pp. 2786-2796, 2005.

[37] E. M. Gatto, V. Parisi, D. P. Converso et al., “The LRRK2 G2019S mutation in a series of Argentinean patients with Parkinson's disease: clinical and demographic characteristics," Neuroscience Letters, vol. 537, pp. 1-5, 2013.

[38] C. Marras, B. Schuele, R. P. Munhoz et al., "Phenotype in parkinsonian and nonparkinsonian LRRK2 G2019S mutation carriers," Neurology, vol. 77, no. 4, pp. 325-333, 2011.

[39] L. L. Kilarski, J. P. Pearson, V. Newsway et al., "Systematic review and UK-based study of PARK2 (parkin), PINK1, PARK7 (DJ1) and LRRK2 in early-onset Parkinson's disease," Movement Disorders, vol. 27, no. 12, pp. 1522-1529, 2012.

[40] W. Regragui, L. Lachhab, R. Razine et al., "Profile of idiopathic Parkinson's disease in Moroccan patients," International Archives of Medicine, vol. 7, no. 1, article 10, 2014.

[41] M. Ehrminger, S. Leu-Semenescu, F. Cormier et al., "Sleep aspects on video-polysomnography in LRRK2 mutation carriers," Movement Disorders, vol. 30, no. 13, pp. 1839-1843, 2015.

[42] C. Pont-Sunyer, A. Iranzo, C. Gaig et al., "Sleep disorders in parkinsonian and nonparkinsonian LRRK2 mutation carriers," PLoS ONE, vol. 10, no. 7, Article ID e0132368, 2015.

[43] D.-W. Li, Z. Gu, C. Wang et al., "Non-motor symptoms in Chinese Parkinson's disease patients with and without LRRK2 G2385R and R1628P variants," Journal of Neural Transmission, vol. 122, no. 5, pp. 661-667, 2015. 
[44] S. Goldwurm, M. Zini, L. Mariani et al., "Evaluation of LRRK2 G2019S penetrance: relevance for genetic counseling in Parkinson disease," Neurology, vol. 68, no. 14, pp. 1141-1143, 2007.

[45] D. Gosal, O. A. Ross, J. Wiley et al., "Clinical traits of LRRK2associated Parkinson's disease in Ireland: a link between familial and idiopathic PD," Parkinsonism and Related Disorders, vol. 11, no. 6, pp. 349-352, 2005.

[46] L. V. Kalia, A. E. Lang, L.-N. Hazrati et al., "Clinical correlations with lewy body pathology in LRRK2-related Parkinson disease," JAMA Neurology, vol. 72, no. 1, pp. 100-105, 2015. 


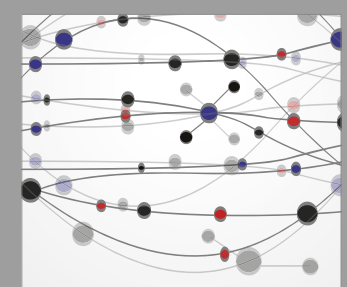

The Scientific World Journal
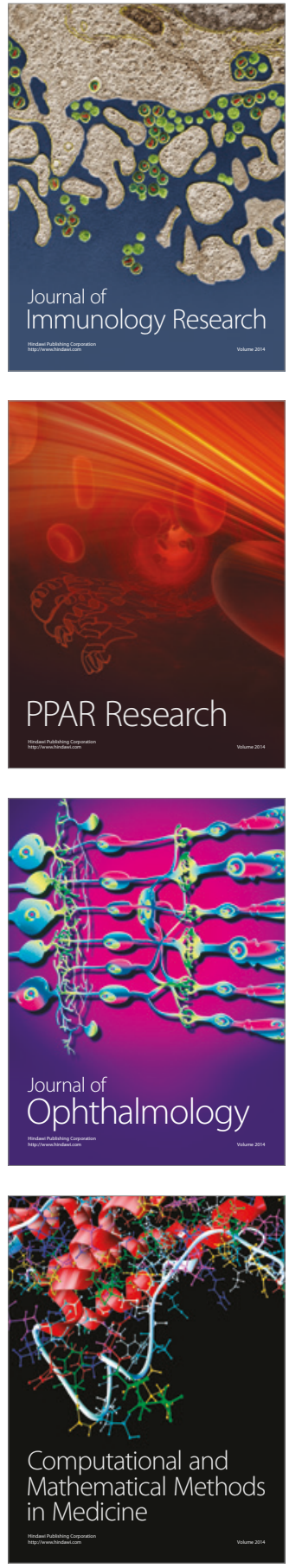

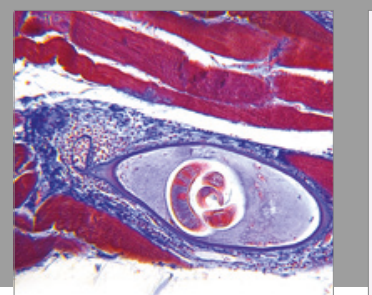

Gastroenterology Research and Practice
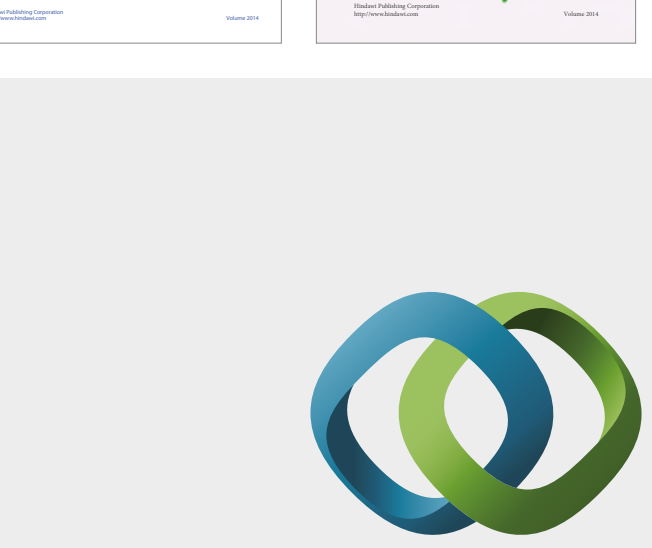

\section{Hindawi}

Submit your manuscripts at

https://www.hindawi.com
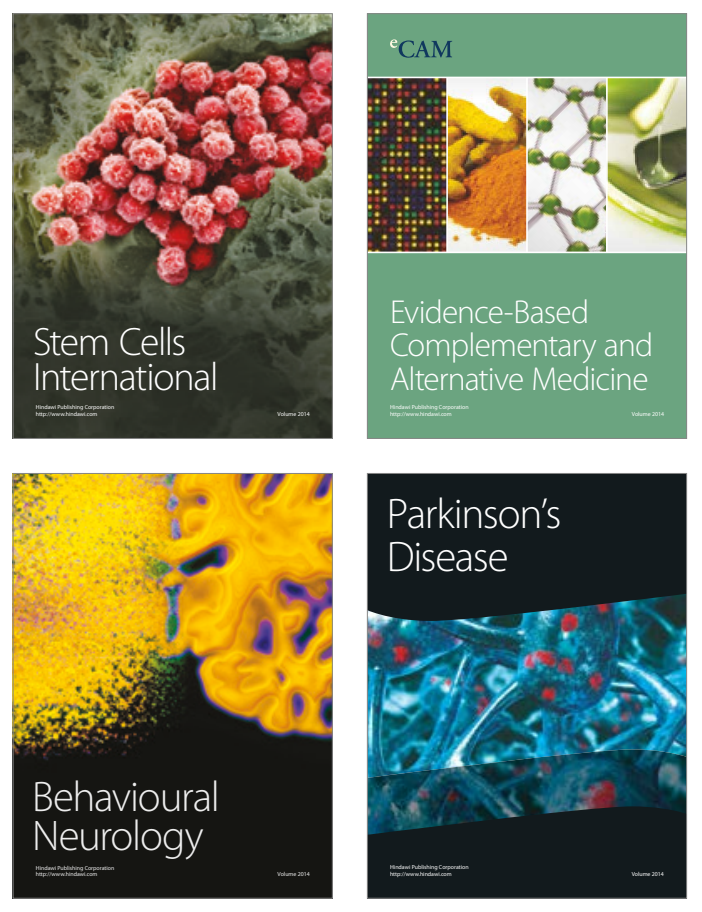
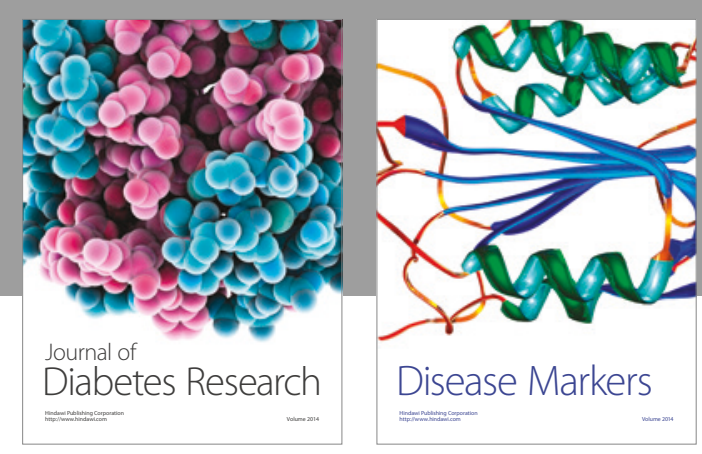

Disease Markers
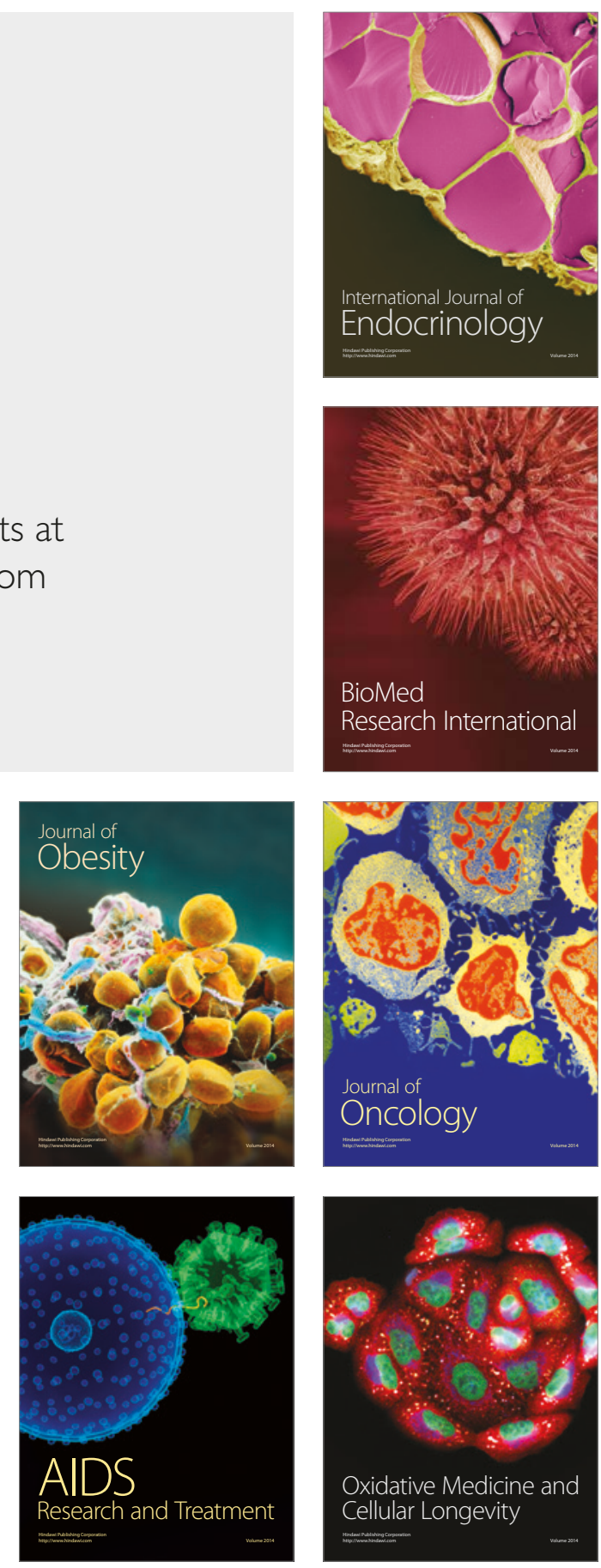